\title{
Low and intermediate mass star yields
}

\section{The evolution of nitrogen abundances}

\author{
M. Gavilán ${ }^{1}$, M. Mollá ${ }^{2}$, and J. F. Buell ${ }^{3}$ \\ 1 Departamento de Física Teórica, Universidad Autónoma de Madrid, 28049 Cantoblanco, Spain \\ e-mail: marta.gavilan@uam.es \\ 2 Departamento de Investigación Básica, C.I.E.M.A.T., Avda. Complutense 22, 28040 Madrid, Spain \\ e-mail: mercedes.molla@ciemat.es \\ 3 Department of Mathematics and Physics, Alfred State College, Alfred, NY 14802, USA \\ e-mail: BuellJF@alfredstate.edu
}

Received 7 June 2005 / Accepted 25 November 2005

\section{ABSTRACT}

Aims. We analyze the impact on the Galactic nitrogen abundances with a new set of low and intermediate mass star yields. These yields contain a significant yield of primary nitrogen from intermediate mass stars.

Methods. We use these yields as an input for a galactic chemical evolution model and study the nitrogen abundances in the halo and in the disc, and compare them with models obtained using other yield sets and with a large amount of observational data.

Results. We find that with these new yields our model reproduces the observed trends adequately. In particular, these yields solve the historical problem of nitrogen evolution, giving the right level of relative abundance N/O by producing a primary component in intermediate mass stars. Moreover, using different evolutionary rates for each radial region of the Galaxy, we can explain the observed $\mathrm{N}$ dispersion.

Key words. galaxies: abundances - galaxies: evolution - galaxies: spirals

\section{Introduction}

Most elements are created in the interiors of stars by nucleosynthesis processes (see Wallerstein et al. 1997, for a review), starting with hydrogen and progressing toward heavy elements. These processes are called primary production. Some elements, however, can be formed from heavier nuclei than the hydrogen originally present in the star. They are called secondary. This is the case for nitrogen, which can be created during the CNO cycle using seeds of original carbon and/or oxygen. From a theoretical point of view, it has been considered that massive stars produce secondary nitrogen (Peimbert 1987), while low and intermediate mass (LIM) stars have mechanisms, like the third dredge-up and the hot bottom burning (HBB) processes, to produce both primary and secondary nitrogen (Edmunds \& Pagel 1978; Alloin et al. 1979). The third dredge-up event is a consequence of the thermal pulses in the star, and transport $\mathrm{C}$ and He to the outer layers. The HBB occurs when the CNO cycle takes place at the base of the convective envelope.

Observationally, there are several open questions about the primary or secondary character of nitrogen that up to now remain unsolved. When $\mathrm{N}$ and $\mathrm{O}$ data are represented as $\log (\mathrm{N} / \mathrm{O})$ vs. $\log (\mathrm{O} / \mathrm{H})$, including the galactic stars, HII regions for the Milky Way Galaxy (MWG), external galaxies (Garnett et al. 1995, 1999; van Zee et al. 1998a; Izotov \& Thuan 1999), and the high redshift data (Pettini et al. 2002; Prochaska et al. 2002; Centurión et al. 2003, and references therein), a clear positive slope appears for abundances larger than $12+\log (\mathrm{O} / \mathrm{H})=$ 7.8-8 dex, which indicates a secondary behavior; but the plot shows a flat slope for low metallicities that can only be explained with a primary component of nitrogen. Taking into account that this flat slope occurs for low abundances, the first idea proposed, shared by some authors (Pagel et al. 1979; Diaz \& Tosi 1986; Dahmen et al. 1995) is that observations would be reproduced if the nitrogen ejected by massive stars were primary, while intermediate mass stars might have both primary and secondary components.

Thus, some authors have tried to look for mechanisms that explain how massive stars could produce primary nitrogen. This is the case of Meynet \& Maeder (2002) who have recently proposed rotation as a possible source of primary nitrogen, since low metallicity stars show a bigger rotation than high metallicity ones. Chiappini et al. (2003a) used these yields in their chemical evolution models, concluding that they are only a lower limit for the primary nitrogen production since the 
HBB is not considered in their calculation. In fact, Chiappini et al. (2005) find that an extra production of $\mathrm{N}$ in low metallicity massive stars by a large factor, between 40 and 200 along the mass range, is necessary to explain the data of very metal-poor halo stars since these yields do not produce a sufficient amount of primary N. Moreover, if the production of primary nitrogen proceeded from massive stars, the left side of the (N/O) vs. $(\mathrm{O} / \mathrm{H})$ plot should not show any scatter. Although some authors claim to observe (Izotov \& Thuan 1999; Pilyugin et al. 2003) this lack of scatter, recent observations from low metallicity objects (Pettini et al. 2002; Prochaska et al. 2002; Centurión et al. 2003; Israelian et al. 2004; Spite et al. 2005) do show a clear dispersion.

Serrano \& Peimbert (1983) have already claimed that a secondary production by intermediate mass stars must exist and suggested that the zero slope may be explained by two factors: 1) a delay in the ejection of $\mathrm{N}$ to the ISM due to the different mean-lifetimes of stars and 2) the gas infall effects. The advantage of taking a delay into account is that the large data scatter can be explained by considering different evolutionary states for each galaxy, so this possibility has been supported by a large number of authors: Vila Costas \& Edmunds (1993); Pilyugin (1992, 1993); van Zee et al. (1998b); Henry et al. (2000). This last group also includes gas flows - infall or/and outflow - and low efficiency for the star formation rate (the equivalent mechanism to produce a delay) in the low evolved regions, in order to reproduce the flat slope in the $(\mathrm{N} / \mathrm{O})$ vs. $(\mathrm{O} / \mathrm{H})$ plot. They conclude that the secondary production of nitrogen should dominate in high metallicity environments, while the primary one should act at low metallicities.

Some new yields for LIM stars were given in Gavilán et al. (2005, hereinafter Paper I), where they were adequately evaluated and calibrated by using them in a Galaxy chemical evolution model. It was shown that the results about $\mathrm{C}$ and $\mathrm{O}$ abundances reproduce the Galactic and Solar Neighborhood data adequately. The purpose of this work is to analyze the impact of these stellar yields on the nitrogen abundances. In particular, we use the same prescriptions of Paper I to check if the contribution to $\mathrm{N}$ given by these yields for LIM stars is sufficient to justify the amount of primary nitrogen the observations point to.

We describe the yields in Sect. 2, analyzing in particular the primary and secondary components of the nitrogen production. In Sect. 3 we briefly describe the chemical evolution model. Section 4 is devoted to the results, and the conclusions are presented in Sect. 5.

\section{Low and intermediate mass yields: the secondary and primary components of nitrogen}

The aim of this work is the study of the nitrogen behavior, using the same set of yields as in Paper I, that we call BU yields. For comparison purposes we also take the LIM stars yields from van den Hoek \& Groenewegen (1997) and Marigo (2001) that we call VG and MA, respectively. The complete table of BU yields was already given in Paper I for five metallicities: $-0.2,-0.1,0.0,+0.1$, and +0.2 , expressed as $\log \left(Z / Z_{\odot}\right)$,

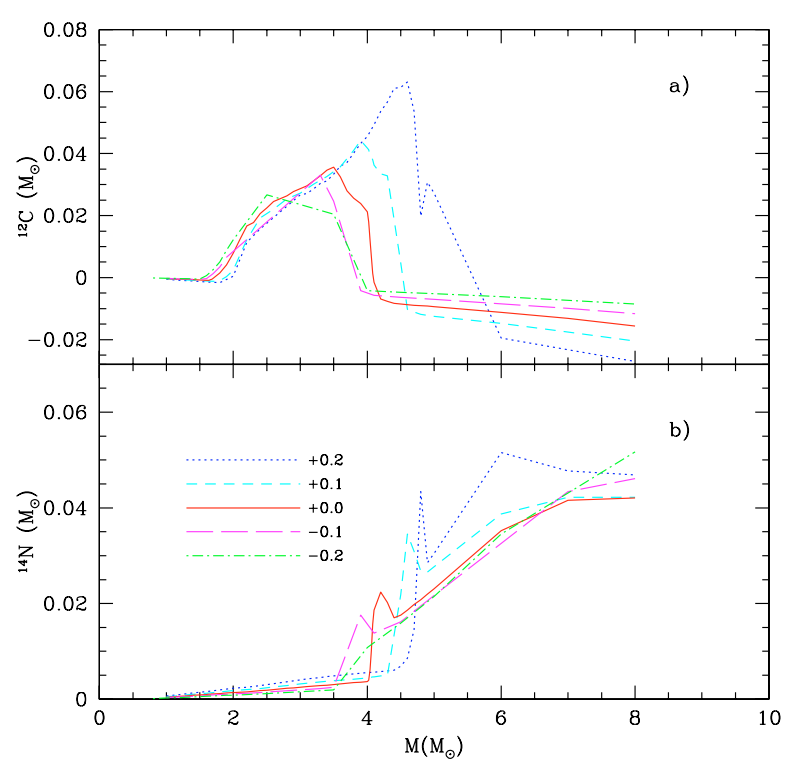

Fig. 1. Total yields (BU) of: a) ${ }^{12} \mathrm{C}$ and b) ${ }^{14} \mathrm{~N}$ produced by LIM stars for different metallicities following label on panel $\mathbf{b}$ ), expressed as $\log \left(Z / Z_{\odot}\right)$.

where solar abundances were taken from Grevesse \& Sauval (1998) $)^{1}$

We summarize the behavior of the carbon and nitrogen yields for LIM stars, as shown in Fig. 1. In panel a) we see that the ${ }^{12} \mathrm{C}$ yield is extremely small for stars with mass lower than $2 M_{\odot}$, since they do not experience third dredge up events. However, stars begin to suffer these kinds of events for smaller masses at lower metallicity. In other words, in the low mass range, the metallicity and the ${ }^{12} \mathrm{C}$ yield are anticorrelated. When the stars have enough mass to undergo HBB, the ${ }^{12} \mathrm{C}$ yield drops abruptly because of the conversion of carbon into nitrogen. The ${ }^{14} \mathrm{~N}$ yield presents a local maximum in the mass range from 3.5 to $5 M_{\odot}$, depending on the metallicity, then decreases before beginning to increase again as a function of stellar mass. The largest amount of nitrogen is produced by stars of intermediate mass because $\mathrm{HBB}$ and the 2nd dredgeup occur only in stars with $M>3.5-5 M_{\odot}$. As the HBB increases the luminosity and the mass-loss rate, stars that suffer this process have shorter TP-AGB lifetimes. The local maximum occurs in the transition between stars with $\mathrm{HBB}$ and those without. The increase at higher masses is due to the shortened time between third dredge-up events. The yields at the lowest masses are due to the 1 st dredge-up.

The most important difference among the used yields resides in the contribution of primary and secondary components of nitrogen by LIM stars. In Fig. 2 we represent the fraction of primary ${ }^{14} \mathrm{~N}$ for the three sets used, as labeled, as a function of mass $\left(M \leq 8 M_{\odot}\right.$, except for MA, for which $\left.M \leq 5 M_{\odot}\right)$. In panel a) we show the results for solar abundances. All of

1 The use of these solar abundances implies that $Z_{\odot}=0.02$. Recently, Asplund et al. (2005) have obtained lower abundances that lead to a value $Z_{\odot}=0.012$. However, these new determinations are still questioned by some authors (Bahcall et al. 2005; Drake \& Testa 2005; Antia \& Basu 2005) because they do not fit the helioseismological constraints. 


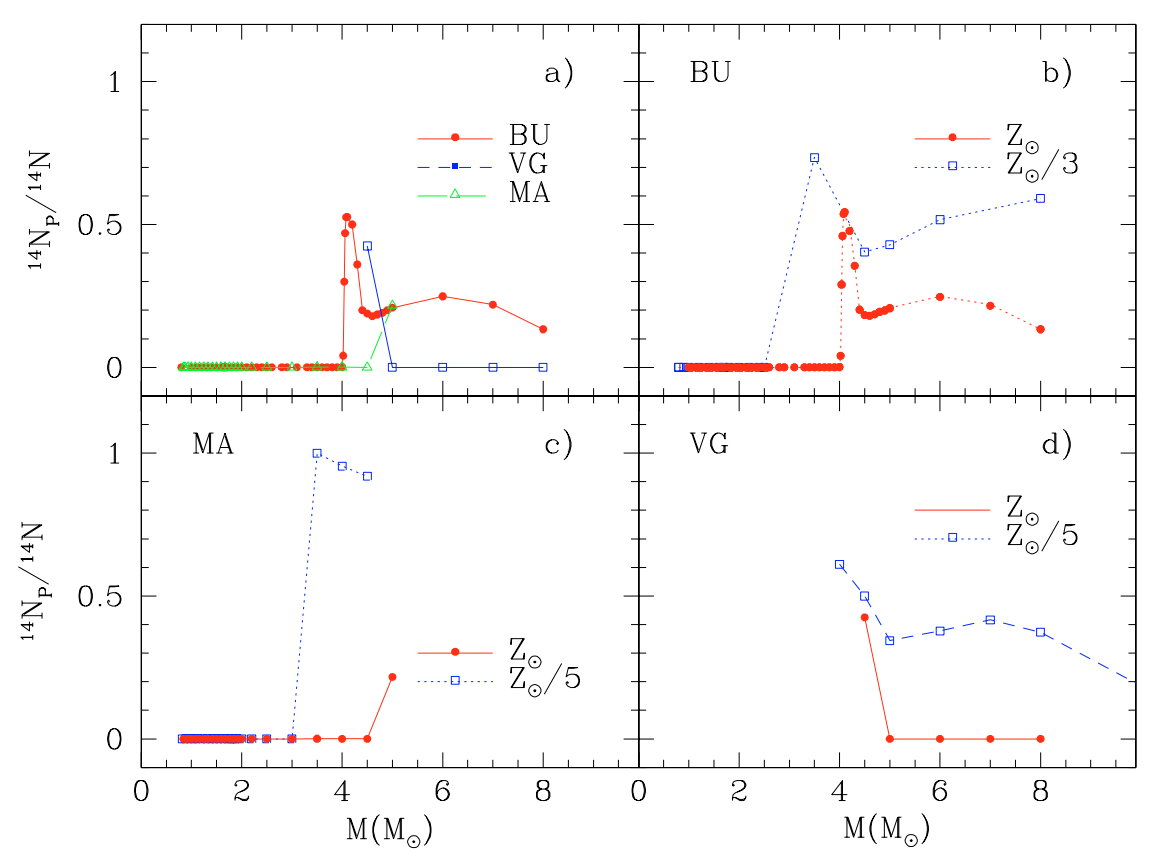

Fig. 2. The ratio of the yield of primary ${ }^{14} \mathrm{~N}$ to the total yield of ${ }^{14} \mathrm{~N}$ as derived from different author sets: BU, VG, and MA. Panel a) a comparison of the solar metallicity yields for the three sets as labeled. Models for two different compositions, as labeled, are represented in panels b), c), and d) for BU, MA, and VG, respectively.

them show a similar behavior with a maximum for masses around 3.5-4 $M_{\odot}$.

We must clear up some points about the components of $\mathrm{N}$. The only difference between primary and secondary nitrogen is the origin of the carbon atom producing it. Although the idea is conceptually clear, it is not so simple to separately compute both components. Thus, although BU and MA give the two components separately for each model, VG do not. These authors, however, show their yields in each phase of stellar evolution. If we consider that all the nitrogen created in the AGB phase is primary, about $\sim 90 \%$ of the N ejected by LIM stars will be primary. This is sometimes assumed when these yields are used. This hypothesis, which we call AGB technique leads to a primary $\mathrm{N}$ component that is excessively large and is not totally adequate.

Let us return to the definition: secondary $\mathrm{N}$ proceeds from the burning of original ${ }^{12} \mathrm{C}$. If a fraction of the original carbon is burned in the pre-AGB phase, it produces secondary $\mathrm{N}$. Sometimes, this gives a negative ${ }^{12} \mathrm{C}$ yield. But, not all the initial carbon is consumed before the AGB phase. If we take a star of $4 M_{\odot}$ of solar abundance as an example, that is, with $X\left({ }^{12} \mathrm{C}\right)=0.28 \times 10^{-2}$, it has an initial ${ }^{12} \mathrm{C}$ mass $m\left({ }^{12} \mathrm{C}\right)=$ $4 M_{\odot} X\left({ }^{12} \mathrm{C}\right)=1.12 \times 10^{-2} M_{\odot}$. The pre-AGB phase carbon yield is $y \mathrm{C} 12_{\text {pre }}=0.300 \times 10^{-4}$, so the mass of this element present in the star before the AGB begins is:

$M\left({ }^{12} \mathrm{C}\right)=y \mathrm{C} 12_{\mathrm{pre}} M_{\mathrm{ini}}+M_{\mathrm{end}} X\left({ }^{12} \mathrm{C}\right)$,

where $M_{\text {end }}$ is the mass of the star at the end of this first phase: $3.95 M_{\odot}$. Therefore, there is a mass $\sim 1.118 \times 10^{-2} M_{\odot}$ of ${ }^{12} \mathrm{C}$, from which $M_{\text {end }} X\left({ }^{12} \mathrm{C}\right) \sim 1.102 \times 10^{-2} M_{\odot}$ corresponds to the original carbon. This implies that a quantity of the original carbon is still available to form nitrogen in the following phases. Thus, a fraction of the total nitrogen produced in the AGB phase (given by the addition of the two values given by VG in their tables denoted AGB yields and final AGB yields) may be secondary. In order to calculate this component from the total AGB yields, we use the fraction, called $r$, between the secondary to the total nitrogen yield, $r={ }^{14} \mathrm{~N}_{\mathrm{S}} /{ }^{14} \mathrm{~N}$. Taking into account that the secondary $\mathrm{N}$ proceeds from the existing carbon used as a seed, we assume that $r$ is equal to the ratio between the old carbon and the new-plusold carbon:

$r=\frac{{ }^{14} \mathrm{~N}_{\mathrm{S}}}{{ }^{14} \mathrm{~N}}=\frac{{ }^{12} \mathrm{C}_{\mathrm{old}}}{{ }^{12} \mathrm{C}_{\mathrm{old}}+{ }^{12} \mathrm{C}_{\text {new }}}$.

This method (hereinafter called $r$ method), may be applied only to stars that suffer the HBB and produce primary ${ }^{14} \mathrm{~N}$, that is, those for which the core mass before the HBB is larger than $M_{\mathrm{HBB}}=0.8 M_{\odot}$, usually stars with $M>3.5-4 M_{\odot}$. Otherwise, the nitrogen yield is totally secondary. The results of VG shown in Fig. 2 proceed from this calculation.

We have then computed the integrated yields of ${ }^{14} \mathrm{~N}$ produced by LIM stars that we present in Fig. 3 as a function of metallicity $Z$. In panel a), we represent the BU results as solid circles on which we have performed a least-squares fit shown by the solid (red) line. This integrated yield for ${ }^{14} \mathrm{~N}$, equivalent to the yield produced by a single stellar population, is located between the two other sets in this panel, showing a similar dependence on $Z$ as $\mathrm{VG}^{2}$ but with lower absolute values.

More significant, however, is how the ratio of primary to total integrated yields, ${ }^{14} \mathrm{~N}_{\mathrm{P}} /{ }^{14} \mathrm{~N}$ depends on metallicity, shown in panel $b$ ). This ratio increases for decreasing metallicity for

2 The integrated yields for VG are slightly different than those obtained by Henry et al. (2000) due to the different initial mass function (IMF) used by us, from Ferrini et al. (1992), and by them, a Salpeter law. 


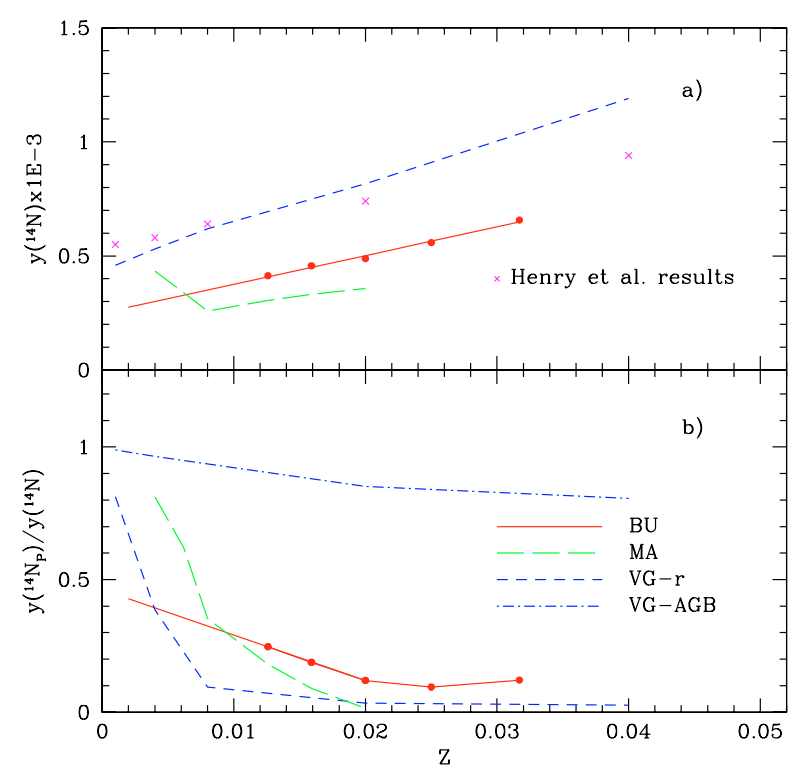

Fig. 3. a) Dependence of the integrated yield of ${ }^{14} \mathrm{~N}$ produced by LIM stars on metallicity for the three yield sets, marked with different symbols as in Fig. 2. The crosses are the results obtained by Henry et al. (2000) for the same VG yields using a Salpeter IMF. b) The ratio between the primary to the total nitrogen yield with the same symbols than in panel a). The two possible techniques to compute the primary component of VG yields are represented as short-dashed line (method $r$ ) and dot-short-dashed line $(\operatorname{method} A G B)$.

all sets, as expected, although the integrated yield for VG is quite different if we consider the AGB technique rather than the $r$ method.

This metallicity effect can be easily explained: low metallicity stars have smaller radii and take longer to reach superwinds, so they have more time to experience third dredge-up events than solar metallicity stars. As a consequence, they have more fresh ${ }^{12} \mathrm{C}$ in their envelopes and can make more primary nitrogen by the $\mathrm{HBB}$ process. On the other hand, due to the lower amount of original carbon, they produce, obviously, less secondary nitrogen. For VG yields the ratio is almost constant at a value of $15 \%$ with the $r$ technique, represented by the shortdashed (blue) line, for metallicities greater then 0.01 , increasing for metallicities lower than this value. While it is $\sim 90 \%$ when the $A G B$ technique, represented by a dot-short-dashed (blue) line, is used, showing a smooth variation with $Z$. It is interesting that the integrated yield for solar abundance in the BU case $^{3}$ is around $20 \%$, very similar to the value computed as necessary by Alloin et al. (1979) two decades ago on the basis of the observations available at that time.

All these considerations indicate that the primary nitrogen appears on a different time scale in the ISM depending on the value of $Z$. The first primary $N$ will be ejected when stars of $8 M_{\odot}$ die (5 for MA), while $Z$ is still low.

${ }^{3}$ We used the same yields set from $Z=0.0126$ for $Z<0.0126$. It is always possible to extrapolate the trend obtained for the other $Z$ sets, which we show with the straight line joining the points corresponding to $Z=0.0126$ and $Z=0.0159$.

\section{The chemical evolution model and its calibration}

\subsection{Description}

The model used in this work is the multiphase chemical evolution model described in Ferrini et al. $(1992,1994)$, in the version presented in Mollá \& Díaz (2005), and in Paper I. For LIM stars, we used the same yields BU as in these two last papers, and for comparison purposes, those from MA and VG. For massive stars we chose Portinari et al. (1998) and Woosley \& Weaver (1995, hereinafter PCB and WW, respectively). We ran different models computed with different combination of yields: $\mathrm{BU}+\mathrm{WW}, \mathrm{VG}+\mathrm{WW}$, and $\mathrm{MA}+\mathrm{PCB}$, which we distinguish as BU, VG, and MA, respectively.

The nitrogen study is usually done by comparing its behavior relative to iron and oxygen, so it is very important to have a careful calibration for these two elements. Oxygen calibration was done in Paper I. The SNIa are the main manufacturer of iron. The yields for type Ia supernova (SNIa) explosions are taken from Iwamoto et al. (1999) and Branch \& Nomoto (1986). The evolution of this element in the model is quasiindependent of the normal stars yields. However, since iron is mainly produced by SNIa, even if its yield is very well known, its abundance is very dependent on the method of computing the rate of these explosions. For this purpose we analyzed the results obtained with different possibilities in order to eliminate, if possible, uncertainties in the iron abundance evolution. This point is relatively important because the Age-metallicity relation and the G-dwarf metallicity distribution are usually used as calibration methods for chemical evolutions models. Furthermore, we go on to compare our results with observed stellar nitrogen abundances, most of which are given as $[\mathrm{N} / \mathrm{Fe}]$, so, we checked that the Iron evolution is adequately reproduced by our models before this comparison be made.

We used three methods to compute the SNIa rates as given by the following authors: the classical one (Matteucci \& Greggio 1986; Ferrini \& Poggianti 1993), the one given by Tornambe (1989), and the last, more recent, described in Ruiz-Lapuente et al. (2000), hereinafter MAT, TOR, and RL, respectively. The first authors estimate the SN rates by using only the initial mass function. We summarize her the method that is well explained in the first two cited works: a proportion of the stellar masses in a given range $\left[M_{\min }-M_{\max }\right]$ will be in binary systems and a fraction of them will develop type Ia supernova. Based on this idea, a mass function for the secondary stars is computed from the original one. Finally the SNIa rate depends on the number of secondary stars that died in each time step, which implies that the time scale for the iron appearing in the ISM is controlled by the mean lifetimes of these secondary stars.

Actually, this time scale does not depend only on the secondary mean lifetimes, since there are other processes that also participate in the conversion of a binary system into a SNIa explosion. It is necessary to take into account the effects of the distances between both stellar components, the orbital velocities, and other parameters to finally obtain the time taken for the system to explode after the moment of its formation. 


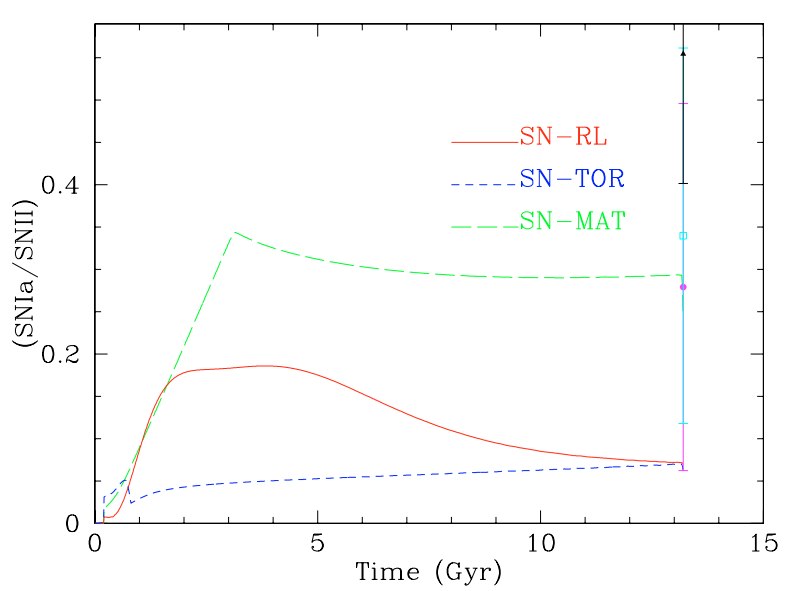

Fig. 4. Evolution of SNIa/SNII rates for MAT, TOR, and RL techniques as labeled in the figure. The observed values for the Galaxy, given by Cappellaro et al. (1999); Cappellaro (2004); Mannucci et al. (2005), are shown with the error bars by the (magenta) full dot, the (cyan) open square, and the (black) triangle, respectively.

Tornambe (1989) performed these calculations for several combinations of possible candidates of binary system or SNIa scenarios (double degenerate, single degenerate, etc.), thereby providing the supernova rate as a function of time normalized for a binary system of $1 M_{\odot}$. All the physical processes and assumptions are included in their calculations, so we only need to include the selected functions in our code and multiply them by the number of binary systems, avoiding the need to compute the secondary and primary initial mass functions necessary in the first method. A similar technique was also performed more recently by Ruiz-Lapuente et al. (2000) who provides us with a numerical table (private communication) with the time evolution of the supernova rates for a single stellar population, computed under updated assumptions about different scenarios and probabilities of occurrence. We computed the supernova rates using the three methods, thus producing three models MAT, TOR, and RL. These different techniques mostly affect the iron abundances, the other elemental abundances being equal for all of them. Therefore, we will compare the three types of SN rate calculations by using only BU yields in the analysis of the iron abundance evolution, as well as in the calibration of the model (next section). We will compare the three set of LIM stars yields when $\mathrm{N}$ is studied, using only the RL technique. Only for the comparison of the relative abundance $[\mathrm{N} / \mathrm{Fe}]$ we will show the nine possible combinations of models.

The main disparity among the three techniques described above resides in the different evolutions of the $\mathrm{SN}$ rate in time. As we see in Fig. 4, MAT is the technique that presents highest values of SNIa/SNII at any time, reaching the maximum at $2.5 \mathrm{Gyr}$. RL has a maximum between 2 and $5 \mathrm{Gyr}$, with values approximately $1 / 2$ or $1 / 3$ of those given by MAT. The TOR model is the only one with low values. Even if it presents a maximum before the first Gyr, this will not be seen in the results because its value is very small. From the first Gyr, SNIa/SNII has positive slope and almost reaches the observed value at the present time. Nevertheless, all of them still are within the error bar given by observations
(Cappellaro et al. 1999; Cappellaro 2004; Mannucci et al. 2005). Note that this value has been reduced for the most recent determinations compared with the oldest ones.

\subsection{Calibration of the model: iron evolution in the Solar vicinity}

The results for iron abundance obtained with these three methods are shown in Fig. 5, the age-metallicity Relation (AMR) and in Fig. 6, the G-Dwarf distribution, for the Solar vicinity. For this comparison we show only BU yields, keeping in mind that the set of yields will have only small effects on this relation. Nevertheless, this model uses WW yields for massive stars and these authors claim that this could produce too much iron, and advised in Timmes et al. (1995) to divide the iron ejections at least by two. In order to calculate how much that WW iron excess is, we calculated four different models for BU yields and RL technique, where the iron production of massive stars is divided by $1,1.5,2$, and 3 . Results are represented in panel a where it is clearly shown that a factor of 2 is a good compromise that we will use in panel b. In this last panel we present the age-metallicity relation for the three SNIa cases, where all of them are in reasonable agreement with data, given their wide dispersion. Although there are small differences between models, it can be seen that the iron appears later and takes a little more time to reach high values when MAT and RL techniques are used than for the TOR SNIa method, but all of them reach the Solar abundance.

Regarding the G-dwarf distribution, represented in Fig. 6, differences appear mainly between TOR and the others because it provides a narrower distribution than the others. The three models are able to reproduce the low metallicity tail without showing any G-dwarf problem.

In Fig. 7 we show the relation between iron and oxygen. As before, in panel a the BU + RL model is presented by varying the massive stars iron ejection. In this case the differences are clearer than in the AMR case. We chose the model Fe/2 that we use for the rest of the paper. In panel $b$ we plot the model results using BU yields with the three SNIa techniques. As in the previous case, the LIM star yields do not change the results because oxygen is ejected by massive stars and iron is mainly produced by SNIa events. In the end, the three models have a very similar behavior.

\section{Results analysis: nitrogen abundances}

We devote this section to analyzing the results obtained with our models for nitrogen abundances and comparing them with the available observational data. We divide these results into four parts: a) the evolution of nitrogen with time for the Solar vicinity (assumed located at a galactocentric distance of $8 \mathrm{kpc}$ ), b) the radial distributions of elements in the disc, c) the relation of $\log (\mathrm{N} / \mathrm{O})$ with the oxygen evolution, and $\mathrm{d})$ the relation of $[\mathrm{N} / \mathrm{Fe}]$ with iron. 


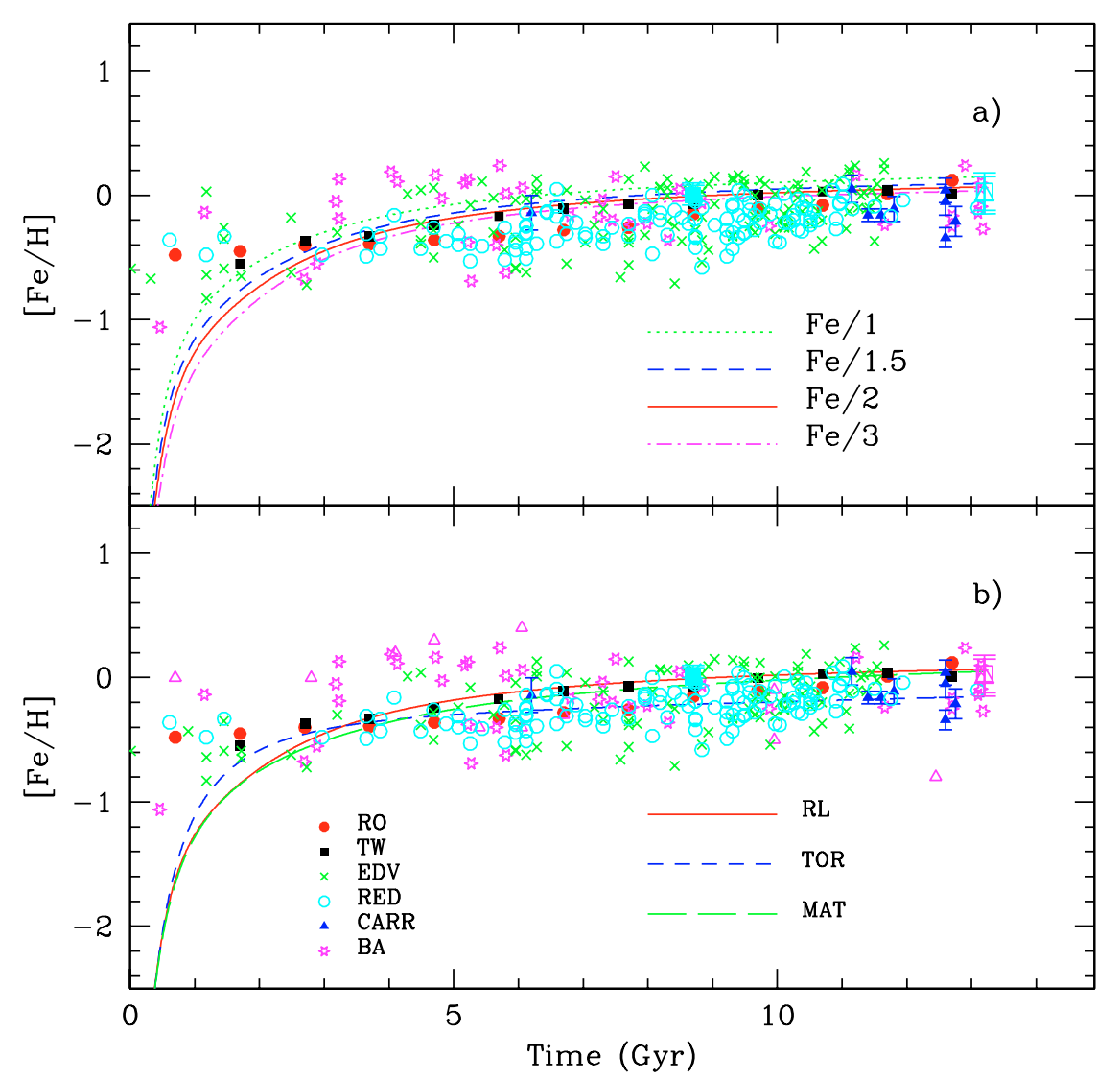

Fig. 5. Age-metallicity relation: a) for the BU and RL model dividing WW iron yield by 1, 1.5, 2, and 2.5. b) using MAT, TOR, and RL SNIa rates with BU yields dividing the WW iron yield by 2. Data are from authors of Table 1 as labeled.

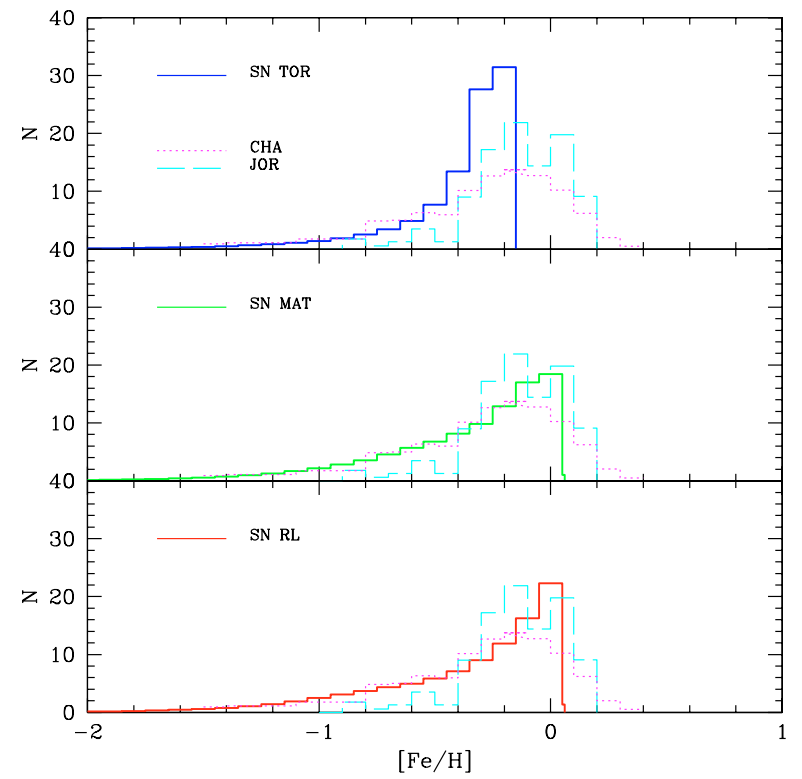

Fig. 6. The G-dwarf distribution in the Solar vicinity. Data are from Chang et al. (2000) (dotted line) and Jørgensen (2000) (long-dashed line)

\subsection{Time evolution of nitrogen}

Figure 8 shows the evolution of nitrogen compared with observations in the Solar region, that is corresponding to objects at a radial galactocentric distance between 7.5 and $9.5 \mathrm{kpc}$. Models BU, VG, and MA are represented by the (red) solid, the (blue) short-dashed, and the (green) long-dashed lines, respectively. Both VG models, following the two possible techniques for calculating the proportion of primary nitrogen, techniques $r$ and $A G B$ described in Sect. 2, yield results indistinguishable for times larger than $1 \mathrm{Gyr}$, so we represent only the results for the first one. In panel a MA and VG models give a higher value than $\mathrm{BU}$, since at the lowest metallicity their nitrogen yield is higher than the corresponding one from BU (see Fig. 2). Then, once an abundance higher than $\sim 0.004$ is reached, the model BU continues increasing smoothly until the present time, reproducing both solar and ISM abundances. The shapes shown by the three models are similar and all of them reproduce both the solar value and the ISM value.

The same kind of information can also be extracted from the relative abundances represented in panel $b$. In panel $b$, we show the time evolution of $\log (\mathrm{N} / \mathrm{O})$. Since there is good agreement in fitting the abundance of oxygen for all models (see Gavilán et al. 2005), the differences in this plot must be due only to the nitrogen production. The disagreement between different models is important for times shorter than $1.5 \mathrm{Gyr}$, when intermediate mass and massive stars are the main contributors and the distinct primary/secondary ratio effects are evident there. Model MA shows a strong increase in the first Gyr due to the primary component, and then it flattens. Model BU out a higher ratio of primary nitrogen than MA for all 


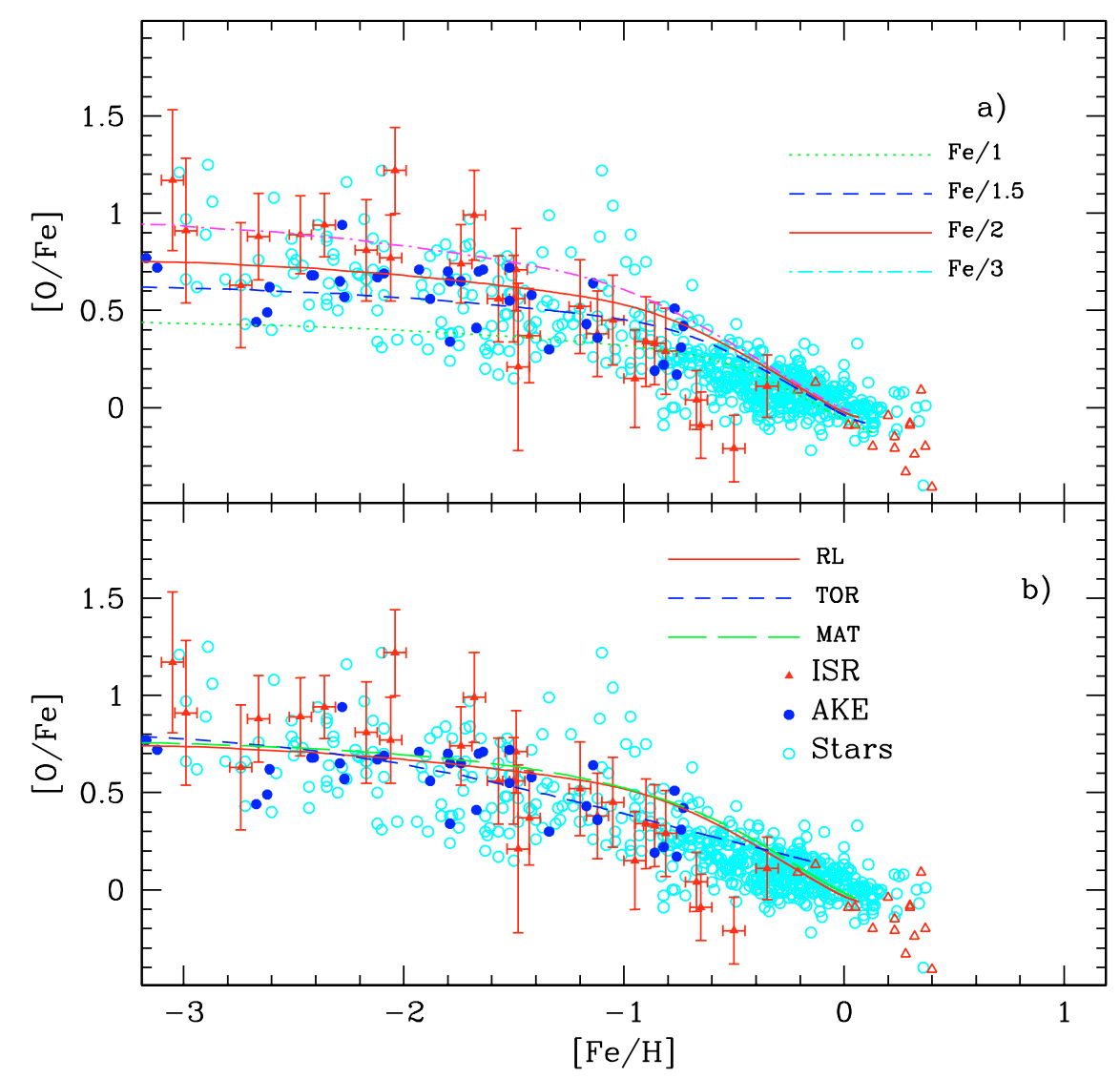

Fig. 7. The relation $[\mathrm{O} / \mathrm{Fe}]$ vs. $[\mathrm{Fe} / \mathrm{H}]$ : a) The model results for $\mathrm{BU}+\mathrm{RL}$ varying WW iron ejection; b) model BU using MAT, TOR, and RL SNIa techniques and BU yields. Open (black) dots are stellar data from authors listed in Table 1, from which we represented the most recent from Israelian et al. (2004) and Akerman et al. (2004) as (red) triangles and full (blue) dots.

$Z$ except for the lowest one, which produces a smoother evolution, and, finally, $\mathrm{N}$ remains below MA. The resulting final $\mathrm{N} / \mathrm{O}$ ratios are similar in both models and agree with observations. The mostly primary behavior of the model VG, mostly when all AGB nitrogen is considered as such, at the lowest $Z$, implies a very strong increase in the abundance at the earliest times. After that, both methods give a smooth slope, reaching an absolute value around -0.5 dex that is higher than the observed Solar abundance.

The good behavior of BU yields is also evident in panel $\mathrm{c}$ where $\log (\mathrm{N} / \mathrm{C})$ is shown. The MA model presents a maximum at the first Gyr, the decrease is due to the higher amount of carbon ejected in that model (see Paper I) so the absolute value at the present time is only marginally reached. The shape of the VG model is similar to the BU one but with a nitrogen excess. All models seem to fit the solar and ISM data, but the model BU is the best one for reproducing the stellar data, and, more importantly, for fitting all of the data simultaneously.

\subsection{Nitrogen abundance in the Galactic disc}

We now explore the radial distributions of nitrogen over the galactic disc, as shown in Fig. 9. The data correspond to HII regions from references as labeled in the figure and to stars from references in Table 1.
The radial distribution is reproduced more or less within the errors by all models. Actually, the shape of the radial distribution is fitted well in all cases, independent of the absolute values, since this is an effect of the ratio infall/SFR along the galactocentric radius, produced by the scenario of our MWG model, and therefore is rather independent of the yields used. However, the observed slope of the radial distribution at the two ends of the disc, in the center and in the outer regions, is a matter of discussion. Thus, Vílchez \& Esteban (1996) claim that the gradients are not as steep in these regions as in the rest of the galactic disc. The same occurs in the inner disc where the most recent data from Smartt et al. (2001) show that the distribution flattens. Our models have been tuned to fit these two sets of data, causing the resulting overall gradient to be smaller than that obtained by other authors. As can be seen in Fig. 9, MA and VG models produce a flatter gradient than has been observed.

In Figs. 9b and 9c, the radial distributions $\log (\mathrm{N} / \mathrm{O})$ and $\log (\mathrm{N} / \mathrm{C})$ are plotted, as they are considered important for the study of different yields. In panel $b$ the radial distribution of $\log (\mathrm{N} / \mathrm{O})$ showed by data presents a clear slope, although there is some data that shows a flatter distribution in the outer regions. A steep radial distribution for N/O is expected because oxygen is produced by massive stars. If nitrogen were ejected by massive stars, its secondary character would cause it to enter the ISM after the oxygen. Instead, if it were ejected by 


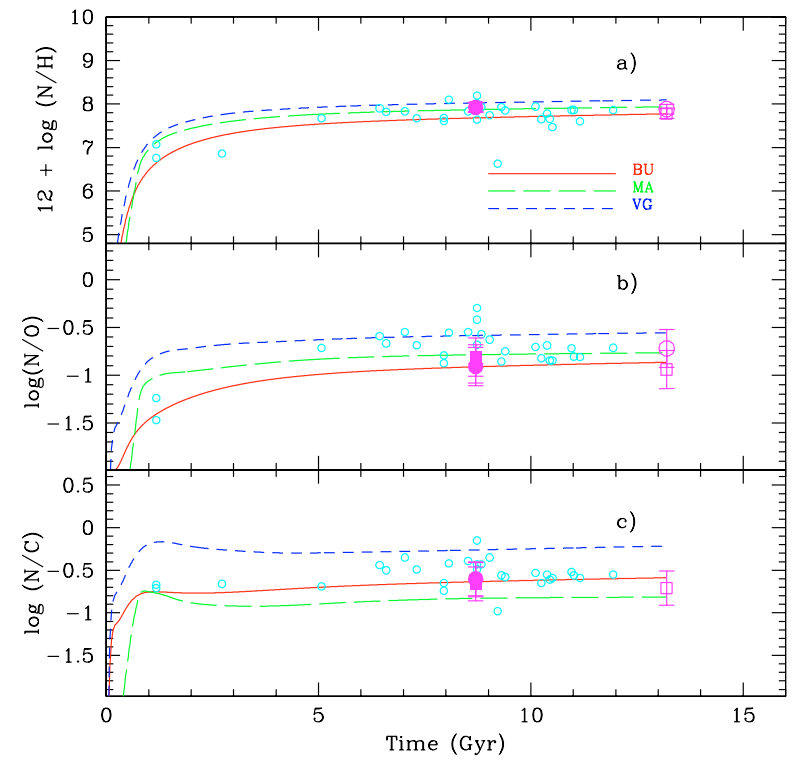

Fig. 8. Time evolution of elemental abundances in the Solar vicinity for nitrogen, as $12+\log (\mathrm{N} / \mathrm{H})$; b) nitrogen over oxygen as $\log (\mathrm{N} / \mathrm{O})$; and c) nitrogen over carbon as $\log (\mathrm{N} / \mathrm{C})$. Solar abundances are the filled symbols from Grevesse \& Sauval (1998) - circle - Holweger (2001) - square - and Asplund et al. (2005) - cross - by assuming an age of 4.5 Gyr for the sun. Empty symbols at $13.2 \mathrm{Gyr}$ are the interstellar medium abundances given by Meyer et al. $(1997,1998)$ - circle and Peimbert (1999) - square. Small open dots are stellar abundances obtained by authors from Table 1, being those located around the Solar vicinity $(7.5<R<9.5 \mathrm{kpc})$. Lines meaning is given in panel a).

intermediate stars, the time needed for their evolution would be larger. Thus, in both cases, the nitrogen appears in the ISM after the oxygen does.

Once again, the large dispersion of the data prevents a clear selection of the best model, however the BU model seems most adequate for reproducing the HII region data from Vílchez \& Esteban (1996) and Fich \& Silkey (1991). The MA model shows a flatter radial gradient than the indicated by observations, with higher absolute values compared to the mean values of data, mostly in the outer disc, and VG models, as before, show higher values than data. The same arguments are also valid when panel $\mathrm{c}$ is analyzed. In this case a slightly negative gradient is shown for $\log (\mathrm{N} / \mathrm{C})$. The small amount of data and the large dispersion prevent the selection of any model as better than the others, although it seems clear that MA remains below most of them as corresponds to the large production of $\mathrm{C}$ and that VG lies on the upper side of the data. It is apparent that the BU model shows a behavior better than VG and MA compared to the data. Once again we stress the importance of using adequate yields to reproduce simultaneously the whole set of data at the same time. The BU yields seem to produce the adequate quantity of $\mathrm{N}, \mathrm{C}$, and $\mathrm{O}$, since the model appears in the zone occupied by data in the three panels simultaneously.

It is necessary to remember that open dots represent stellar abundances. We tried to select only those corresponding to young stars, but we do not know the age of the complete set of stars with available data. In this case we preferred to use the available abundances; thus, it is possible that some data does not correspond to stars young.

\subsection{Nitrogen vs. iron}

As the iron evolution may have also an influence over the model results, we represented the relation between nitrogen and iron, Fig. 10, with a different panel, a, c, and e, for each set of yields, MA, BU, and VG, respectively. The three possible methods of computing the SNIa, RL, TOR, and MAT are shown in each panel. The first thing we observe is that the effects of the different SNIa techniques are almost indistinguishable. Therefore, the main features of each model at those metallicities are due to yields. In other words, we may analyze the behavior of the nitrogen that corresponds to each yield set disregarding the accuracy in the SNIa calculations.

All results agree in the sense that the first nitrogen to be ejected is secondary, as due to the massive stars, so the initial slope is positive and large - although this behavior is not shown in the figure because it occurs when iron abundances are lower than -5 dex - but they differ when the slope begins to change. When the $\mathrm{N}$ ejected by LIM stars appear, there is a strong increase due to the change from a secondary to a primary behavior. In MA yields, LIM stars eject less primary nitrogen and later, since it is ejected as secondary for stars up to $5 M_{\odot}$. Then the main contributors to primary $\mathrm{N}$ are the stars with masses between $2 M_{\odot}$ and $3 M_{\odot}$. For this reason the slope does not change until it reaches $[\mathrm{Fe} / \mathrm{H}]=-2.5$, the moment at which these stars begin to die. When BU yields are used, the trend changes earlier in the evolution due to the contribution of the primary nitrogen ejected by stars in the range $4-8 M_{\odot}$. As their lives are so brief, the ejection occurs at $[\mathrm{Fe} / \mathrm{H}]=-4$. From then, the slope is close to zero, the signature of primary nitrogen. The case of VG behaves like BU.

The data dispersion is so great that all the models lie in the data area, regardless of their large discrepancies, although the region of the metal-rich objects $([\mathrm{Fe} / \mathrm{H}]>-1.5)$ is particularly well fitted in panel $\mathrm{c}$ by Model BU. It is necessary to use the very low metallicity data to clarify which model works better. In fact, the most recent observations from Israelian et al. (2004) and Spite et al. (2005) show a slope flatter than before, even with a negative slope, which is more consistent with our model BU than with the model obtained with MA yields. This last model might be considered acceptable when the available low-metallicity data were only those from Carbon et al. (1987); but when using the new determinations of $\mathrm{N}$ abundances for this kind of object, the conclusion is that BU reproduces the generic trend of data better than the other models.

It is also necessary to take into account that most of the metal-poor objects do not belong to the disc but to the halo. In this way, we represent the halo model results for the zone located over the disc at a galactocentric distance equal to $8 \mathrm{Kpc}$, the right panels $b$, d, and $f$ of Fig. 10. We see that the trend shown by the recent observations from Israelian et al. (2004) and Spite et al. (2005) is more compatible with BU and VG than MA. 


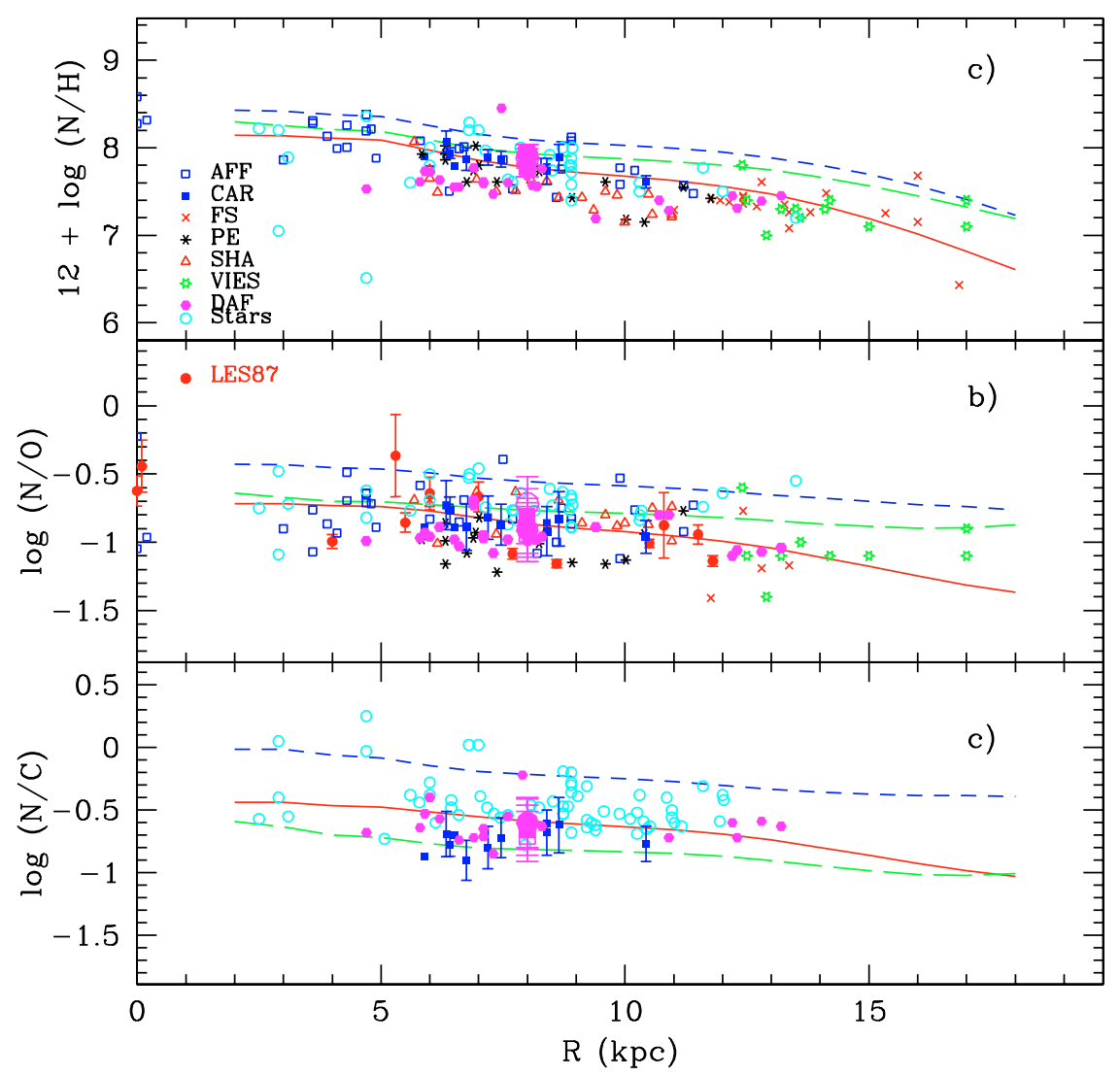

Fig. 9. Radial distributions of nitrogen abundances, a) as $12+\log (\mathrm{N} / \mathrm{H}), \mathbf{b})$ as $\log (\mathrm{N} / \mathrm{O})$ and $\mathbf{c})$ as $\log (\mathrm{N} / \mathrm{C})$ for the same models as in the previous figure. Stellar data - open dots - are taken from the authors given in Table 1, including the most recent ones from Daflon \& Cunha (2004) marked separately. Galactic HII region abundances from Peimbert (1979, PE), Shaver et al. (1983, SHA), Lester et al. (1987, LES87), Fich \& Silkey (1991, FS), Vílchez \& Esteban (1996, VIES), Afflerbach et al. (1997, AFF), Esteban et al. (1999, 2005), and Carigi et al. (2005, the three last ones as CAR) are included as labelled. The Solar and interstellar abundances have the same symbol than in Fig. 8.

\subsection{Nitrogen vs. oxygen}

Finally, we show in Fig. 11 the classical and well-known graph of the relative abundance of $\mathrm{N}$ vs. $\mathrm{O}$ as $\log (\mathrm{N} / \mathrm{O})$ vs. $12+\log (\mathrm{O} / \mathrm{H})$. We show the results of the computed models for the Solar neighborhood. Model BU reproduces the expected behavior of $\mathrm{N}$ when all data are taken into account. Not only is the level of $\mathrm{N}$ adequate but the shape is also smoother than the one shown by the other two models. The observed trend at low metallicity can be reproduced well with BU yields because they have the appropriate primary to secondary ratio, and the adequate integrated nitrogen yields. The MA yields also have a primary nitrogen component, but the integrated nitrogen yield has a metallicity dependence in the opposite way as BU for the lowest $Z$, so the trend shown by the data can not be reproduced. Both VG models have the right shape and are almost the same for $12+\log (\mathrm{O} / \mathrm{H}) \geq 8$. The problem is that the integrated yields are high. It would be necessary to change the input parameters, such as the infall rate or the efficiencies to form stars, in order to fit the solar abundances. In that case, probably, other data will not be reproduced. Only the BU model shows the correct shape and adequate absolute abundances simultaneously. For comparison purposes, we have also shown the resulting model using VG yields but assuming that the Nitrogen is completely secondary.
If the smooth behavior shown by oxygen abundances lower than 8 dex were caused by massive stars, the data dispersion would be smaller than observed. A problem arises when the metal-poor objects (Israelian et al. 2004; Spite et al. 2005) are included in the figure, as can be seen in Fig. 11. Some values follow the described trend over the flat line, but some lower abundances exist that are around $\log (\mathrm{N} / \mathrm{O}) \sim-2$. This behavior is not compatible with a primary component proceeding only from massive stars.

We show the evolution given by the BU model in Fig. 12 for four different radial regions of the Galaxy: two inner $(\sim 2$ and $4 \mathrm{kpc})$ more evolved regions, the solar vicinity $(\sim 8 \mathrm{kpc})$, and an outer one $(\sim 18 \mathrm{kpc})$ where the evolution takes place slowly. In panel a we show the results for the halo zones and in panel $b$ for the disk regions. In both panels we have included the stellar data for the MWG. The numbers on the graph indicate the evolutionary time, in million years, that corresponds to that points of the line. This is necessary because the $12+\log (\mathrm{O} / \mathrm{H})$ value is not the same for each radius at the same value of time.

The halo regions have similar evolutions independent of their distance to the center of the Galaxy. All of them reproduce the recent data from Israelian et al. (2004) and Spite et al. (2005), obtained for $5.5<12+\log (\mathrm{O} / \mathrm{H})<8$ well, while the disk regions fit the stellar data of the disk obtained 
Table 1. References for CNO stellar abundances used for the comparison with model results.

\begin{tabular}{|c|c|c|c|c|c|c|}
\hline Reference & $\mathrm{Fe}$ & $\mathrm{C}$ & $\mathrm{N}$ & $\mathrm{O}$ & $\mathrm{R}$ & Age \\
\hline Akerman et al. (2004) (AKE) & $\mathrm{X}$ & $\mathrm{X}$ & - & $\mathrm{X}$ & - & - \\
\hline Barbuy (1988) & $\mathrm{X}$ & - & - & $\mathrm{X}$ & - & - \\
\hline Barbuy \& Erdelyi-Mendes (1989) & $\mathrm{X}$ & - & - & $\mathrm{X}$ & - & - \\
\hline Barry (1988) (BA) & $\mathrm{X}$ & - & - & - & - & $\mathrm{X}$ \\
\hline Boesgaard et al. (1999) & $X$ & - & - & $X$ & - & - \\
\hline Carbon et al. (1987) (CARB) & $\mathrm{X}$ & $\mathrm{X}$ & $X$ & - & - & - \\
\hline Carraro et al. (1998) (CARR) & $\mathrm{X}$ & - & - & - & - & $\mathrm{X}$ \\
\hline Carretta et al. (2000) & $X$ & $\mathrm{X}$ & $\mathrm{X}$ & $\mathrm{X}$ & - & - \\
\hline Cavallo et al. (1997) & $\mathrm{X}$ & - & - & $\mathrm{X}$ & - & - \\
\hline Chen et al. (2000) & $\mathrm{X}$ & - & - & $\mathrm{X}$ & - & $\mathrm{X}$ \\
\hline Clegg et al. (1981) & $\mathrm{X}$ & $\mathrm{X}$ & $\mathrm{X}$ & $\mathrm{X}$ & - & - \\
\hline Daflon \& Cunha (2004) (DAF) & - & $\mathrm{X}$ & $\mathrm{X}$ & $X$ & $\mathrm{X}$ & - \\
\hline Depagne et al. (2002) & $\mathrm{X}$ & $\mathrm{X}$ & $\mathrm{X}$ & $\mathrm{X}$ & - & - \\
\hline Ecuvillon et al. (2004) & $\mathrm{X}$ & - & $\mathrm{X}$ & - & - & - \\
\hline Edvardsson et al. (1993) (EDV) & $\mathrm{X}$ & - & $\mathrm{X}$ & $\mathrm{X}$ & $\mathrm{X}$ & $\mathrm{X}$ \\
\hline Gustafsson et al. (1999) & - & $\mathrm{X}$ & - & - & $\mathrm{X}$ & $\mathrm{X}$ \\
\hline Friel \& Boesgaard (1990) & $\mathrm{X}$ & $\mathrm{X}$ & - & - & - & - \\
\hline Gratton et al. (2000) & $X$ & $\mathrm{X}$ & $\mathrm{X}$ & $\mathrm{X}$ & - & - \\
\hline Gummersbach et al. (1998) & - & $\mathrm{X}$ & $\mathrm{X}$ & $\mathrm{X}$ & $\mathrm{X}$ & - \\
\hline Israelian et al. $(1998,2001)$ & $X$ & - & - & $X$ & - & - \\
\hline Israelian et al. (2004) (ISR) & $\mathrm{X}$ & - & $\mathrm{X}$ & $\mathrm{X}$ & - & - \\
\hline Laird (1985) & $\mathrm{X}$ & $\mathrm{X}$ & $\mathrm{X}$ & - & - & - \\
\hline Meléndez et al. (2001), & $X$ & $\mathrm{X}$ & - & - & - & - \\
\hline \multicolumn{7}{|l|}{ Meléndez \& Barbuy (2002) } \\
\hline Mishenina et al. (2000) & $X$ & - & - & $X$ & - & - \\
\hline Nissen (2002); Nissen et al. (2002) & $\mathrm{X}$ & - & - & $\mathrm{X}$ & - & - \\
\hline Reddy et al. (2003) (RED) & $\mathrm{X}$ & $\mathrm{X}$ & $\mathrm{X}$ & - & - & $\mathrm{X}$ \\
\hline Rocha-Pinto et al. (2000b,a) (RO) & $X$ & - & - & - & - & $\mathrm{X}$ \\
\hline Rolleston et al. (2000), & $\mathrm{X}$ & $\mathrm{X}$ & $\mathrm{X}$ & $\mathrm{X}$ & $\mathrm{X}$ & - \\
\hline \multicolumn{7}{|l|}{ Smartt \& Rolleston (1997), } \\
\hline \multicolumn{7}{|l|}{ Smartt et al. (2001) } \\
\hline Shi et al. (2002) & $X$ & $\mathrm{X}$ & $\mathrm{X}$ & - & - & - \\
\hline Smith et al. (2001) & $X$ & - & - & $X$ & - & - \\
\hline Spite et al. (2005) (SPI) & $\mathrm{X}$ & $\mathrm{X}$ & $\mathrm{X}$ & $\mathrm{X}$ & - & - \\
\hline Tomkin \& Lambert (1984), & $X$ & $X$ & $\mathrm{X}$ & $X$ & - & - \\
\hline \multicolumn{7}{|l|}{ Tomkin et al. (1986, 1995), } \\
\hline Twarog (1980) (TW) & $X$ & - & 一 & 一 & - & $X$ \\
\hline Westin et al. (2000) & $X$ & $X$ & $\mathrm{X}$ & $\mathrm{X}$ & - & - \\
\hline
\end{tabular}

for $12+\log (\mathrm{O} / \mathrm{H})>8$. Their evolutionary tracks, however, are very different, corresponding to their distinct input parameters (infall rates, initial gas masses, efficiencies to form stars,etc.) which are translated into very different star-formation histories. Thus, the dispersion of the MWG data can be explained on the basis of a primary production of nitrogen from LIM stars, (higher for the lowest metallicities) and with different star formation efficiencies in the different regions.

We represent the same results for the disk regions in Fig. 13 compared with data referring to Galactic HII regions taken from the same authors as in Fig. 11, but without limiting the possible galactocentric distance. Other galaxies data (Garnett et al. 1995, 1999; van Zee et al. 1998a; Izotov \& Thuan 1999) are also shown. The large open triangle is the recent estimate obtained from Izotov et al. (2005) for the galaxy with the lowest metallicity known. We have also added the DLA objects data from Pettini et al. (2002); Prochaska et al. (2002); Centurión et al. (2003) as solid points. The disk regions evolve in good agreement with all of them, showing a steeper evolution for the inner regions, while the outer one shows a very flat evolution with a high and constant value $\log (\mathrm{N} / \mathrm{O}) \sim-1.5 \mathrm{dex}$, similar to the behavior of dwarf galaxies. These results suggest that the observed dispersion in this kind of plot, when other galaxies data (such as dwarf or DLA galaxies) are included, might be reproduced if different star formation histories have occurred in different galaxies. This argument has already been invoked by other authors, in particular by Henry et al. (2000) and Prantzos (2003). It was even demonstrated by Pilyugin et al. (2003), who analyzed data for different radial regions in spiral galaxies and showed the changes of the evolutionary track in the plane $\mathrm{N} / \mathrm{O}$ vs. $\mathrm{O} / \mathrm{H}$ for each one of them. It is evident that this kind of behavior may be represented by our models and that the new yields may reproduce the whole set of data better than other yields. In fact, these yields have already been used in a grid of chemical evolution models for a large number of theoretical galaxies (Mollá \& Díaz 2005). A discussion about the resulting N/O abundances and its possible dispersion for different objects may be found in Mollá et al. (2006).

This figure and the behavior of $\mathrm{C} / \mathrm{O}$ vs. $\mathrm{O} / \mathrm{H}$, as shown in Paper I, are the main clues to the present yields are the most adequate for representung the evolution of galaxies. The production of carbon by LIM stars is sufficient for obtaining an increase in $\mathrm{C} / \mathrm{O}$ without the need to invoke mass loss by massive star winds, and the N/O behavior may be reproduced with different star formation efficiencies due to the adequate level of the primary component produced by LIM stars and to the right dependence of this component with $Z$.

\section{Conclusions}

Our conclusion can be summarized as follows:

1. The primary component of nitrogen, necessary to explain the trend of $\mathrm{N} / \mathrm{O}$ with $\mathrm{O} / \mathrm{H}$, may be mostly produced by LIM stars and adequately fits all the data, including the observed dispersion. In this way the integrated yield produced by LIM stars must be directly proportional to $Z$, while the ratio $N_{\mathrm{P}} / N_{\text {tot }}$ must increase for $Z$ decreasing. A primary component, larger for lowest metallicities, has an important effect on explaining low abundance range data.

2. The dependence of the $\mathrm{N}$ yield on stellar mass would have a maximum around 5-6 $M_{\odot}$, while the primary component shows an other around 3.5-5 $M_{\odot}$. This constrains the time where these contributions are important for the evolution.

3. The high dispersion of N/O data for low and high metallicity galactic regions may be explained with these yields as we demonstrated with the models for the radial regions of the disc with different star formation histories. Our 


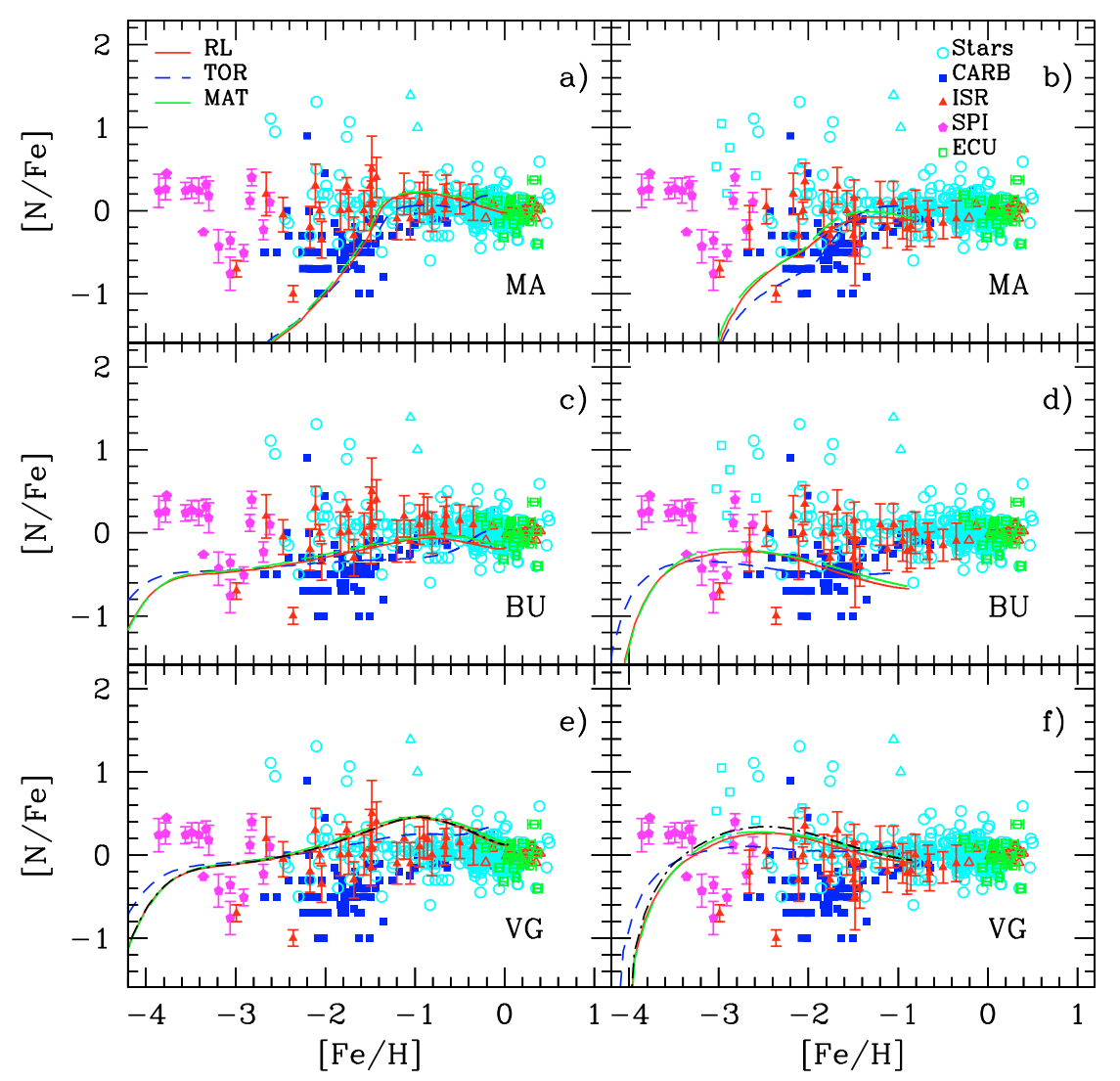

Fig. 10. The relative abundances of $[\mathrm{N} / \mathrm{Fe}]$ vs. the iron abundance $[\mathrm{Fe} / \mathrm{H}]$ for the Solar region. The panels on the left show the disc evolution and the panels on the right show the halo evolution. In each panel, the evolution obtained with three different methods to compute the SN-Ia rates are shown as labeled in panel a). Data are taken from the references listed in Table 1 marking the most recent ones from Israelian et al. (2004); Ecuvillon et al. (2004) and Spite et al. (2005) separately in panel b).

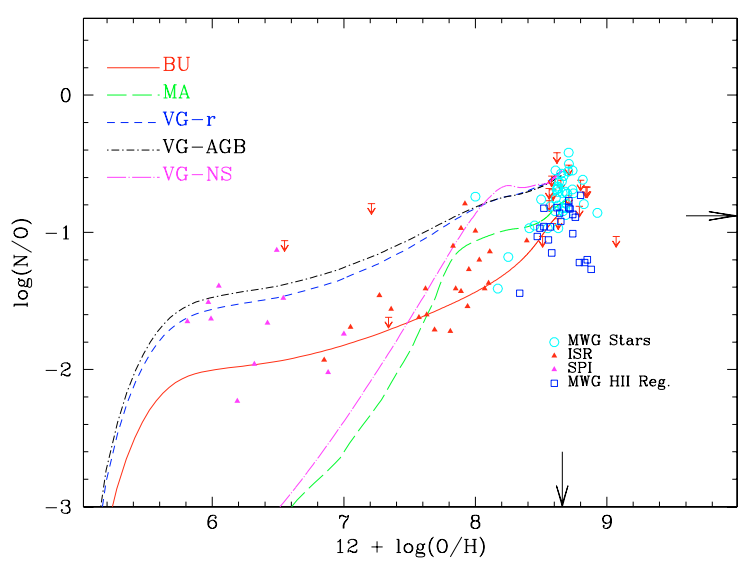

Fig. 11. The relative abundances of $\log (\mathrm{N} / \mathrm{O})$ vs. the Oxygen abundance as $12+\log (\mathrm{O} / \mathrm{H})$ for the Solar neighborhood (lines as shown in the plot). Stellar abundances are the open dots from Table 1, from which we markthose from Israelian et al. (2004) and Spite et al. (2005) separately as (red and magenta) full triangles. Galactic HII region data in the Solar region $(7.5<R(\mathrm{kpc})<9.5$ are from Peimbert (1979), Shaver et al. (1983), Fich \& Silkey (1991), Vílchez \& Esteban (1996), Tsamis et al. (2003), and Carigi et al. (2005). The meaning of symbols is given in the figure. The solar position is marked with arrows.

findings support the previous results from Henry et al. (2000) and Prantzos (2003) obtained using VG yields, but we claim that it is easier to reproduce the whole data set when BU yields are used. Our model for MWG is consistent with the data.

4. These results seem to suggest that models with differences in the star formation histories for different types of galaxies, such as those calculated in Mollá \& Díaz (2005), might produce final abundances with a high dispersion, in agreement with the observed one when dwarf galaxies or DLA galaxies are also included in a plot $\mathrm{N} / \mathrm{O}-\mathrm{O} / \mathrm{H}$, such as we will show in Mollá et al. (2006).

5. The halo and the disc have different evolutions like in the model from Chiappini et al. (2003b). The set of stellar data $[\mathrm{C} / \mathrm{Fe}],[\mathrm{N} / \mathrm{Fe}]$, and $[\mathrm{N} / \mathrm{C}]$ may be divided into two trends. The first one is reproduced by our disc models, while the second one is well-fitted by our halo results.

6. Due to the primary N component of BU yields, and since the intermediate stars have short lifetimes, it is possible to produce high $[\mathrm{N} / \mathrm{Fe}]$ abundances even at low metallicities in perfect agreement with the recent halo stars data obtained by Israelian et al. (2004); Cayrel et al. (2004) and Spite et al. (2005).

7. We claim that the simultaneous fit of the whole set of CNO abundances with only one model for the MWG is not an easy task. We may reproduce the observed trends reasonably well with BU yields combined with yields from WW with our multiphase chemical evolution model. 


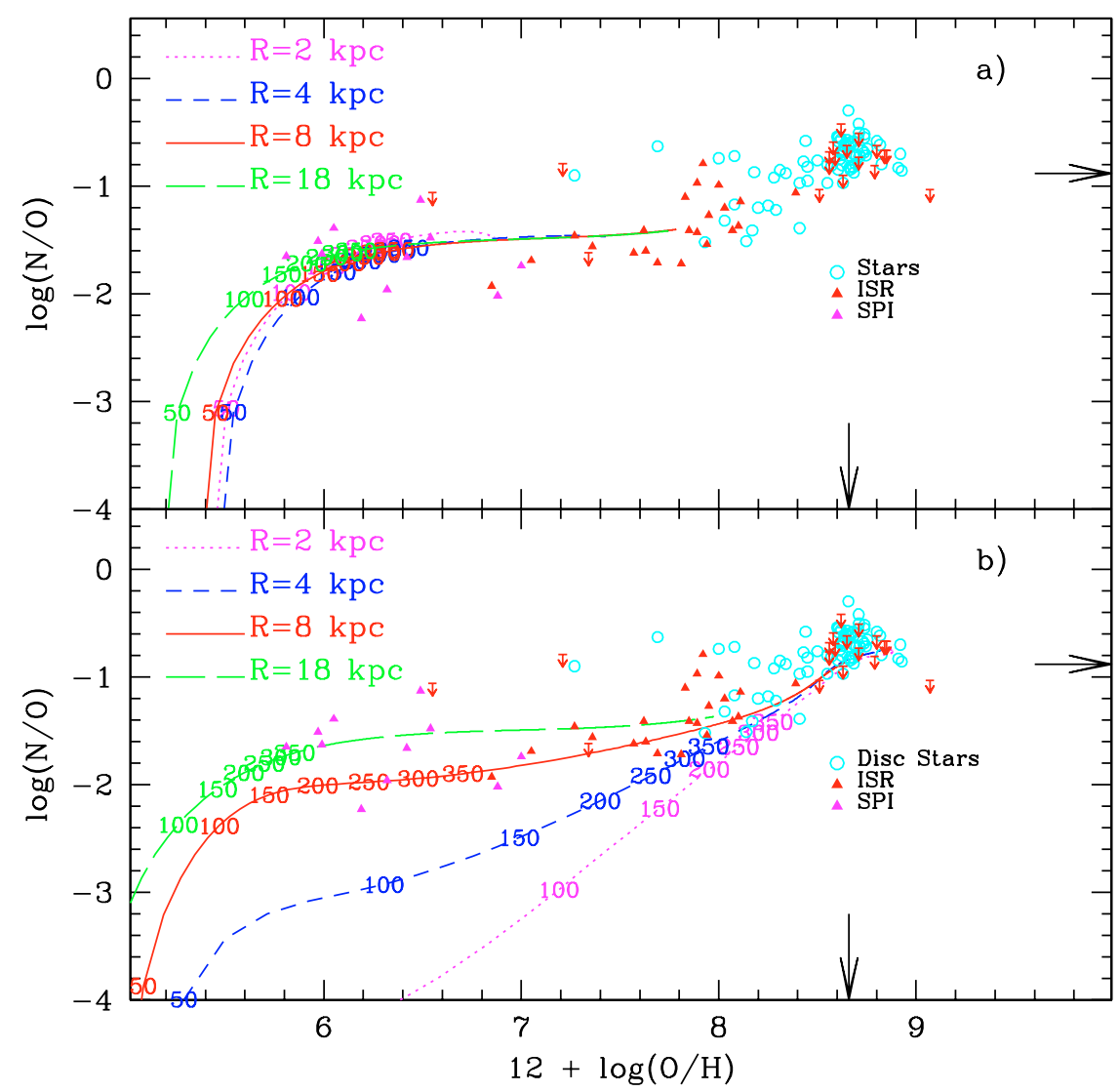

Fig. 12. The relative abundances of $\log (\mathrm{N} / \mathrm{O})$ vs. the Oxygen abundance as $12+\log (\mathrm{O} / \mathrm{H})$ for the halo - upper panel - and for the disc lower panel - for four different galactocentric regions: two inner regions located at 2 and $4 \mathrm{kpc}$, dotted and short dashed lines; a solar region, solid line; and an outer region, long dashed line. Data refering to Galactic stars are from the same authors as cited in Table 1. The meaning of symbols is given in the figure. The solar position is marked with arrows.

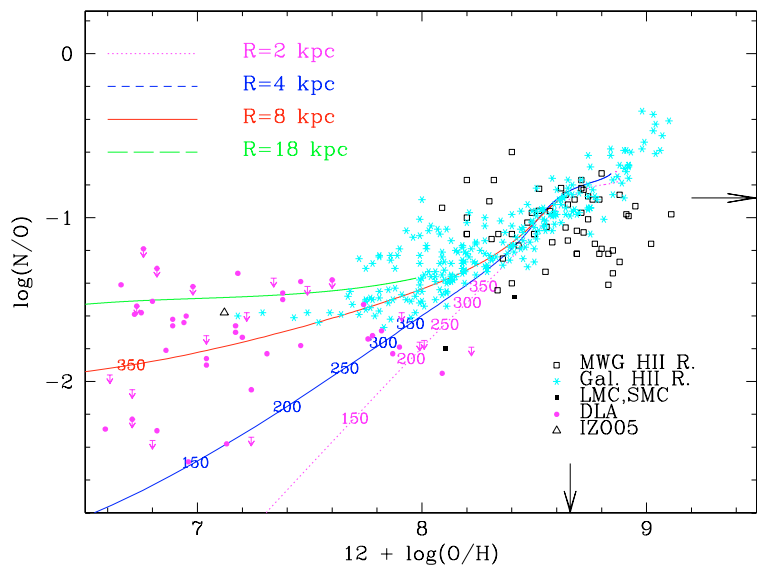

Fig. 13. The relative abundances of $\log (\mathrm{N} / \mathrm{O})$ vs. the Oxygen abundance as $12+\log (\mathrm{O} / \mathrm{H})$ for the same disk regions as the previous figure, compared with data referring to Galactic and extragalactic HII regions and DLA objects data. The meaning of symbols is given and the solar position is marked with arrows.

Acknowledgements. This work was partially supported by the Spanish PNAYA project AYA2004-8260-C03-03. We acknowledge Pilar Ruiz-Lapuente for her personal contribution in the SNIa rates data. We also thank José Manuel Vílchez for his valuable suggestions and the referees, Leonid S. Pilyugin and Angeles I. Díaz, for their comments that have greatly improved this paper.

\section{References}

Afflerbach, A., Churchwell, E., \& Werner, M. W. 1997, ApJ, 478, 190 Akerman, C. J., Carigi, L., Nissen, P. E., Pettini, M., \& Asplund, M. 2004, A\&A, 414, 931

Alloin, D., Collin-Souffrin, S., Joly, M., \& Vigroux, L. 1979, A\&A, 78, 200

Antia, H. M., \& Basu, S. 2005, ApJ, 620, L129

Asplund, M., Grevesse, N., \& Sauval, A. J. 2005, in Cosmic Abundances as Records of Stellar Evolution and Nucleosynthesis, ASP Conf. Ser., 336, 25

Bahcall, J. N., Serenelli, A. M., \& Basu, S. 2005, ApJ, 621, L85

Barbuy, B. 1988, A\&A, 191, 121

Barbuy, B., \& Erdelyi-Mendes, M. 1989, A\&A, 214, 239

Barry, D. C. 1988, ApJ, 334, 436

Boesgaard, A. M., King, J. R., Deliyannis, C. P., \& Vogt, S. S. 1999, AJ, 117, 492

Branch, D., \& Nomoto, K. 1986, A\&A, 164, L13

Cappellaro, E. 2004, Mem. Soc. Astron. Ital., 75, 206

Cappellaro, E., Evans, R., \& Turatto, M. 1999, A\&A, 351, 459

Carbon, D. F., Barbuy, B., Kraft, R. P., Friel, E. D., \& Suntzeff, N. B. 1987, PASP, 99, 335

Carigi, L., Peimbert, M., Esteban, C., \& García-Rojas, J. 2005, ApJ, 623,213 
Carraro, G., Ng, Y. K., \& Portinari, L. 1998, MNRAS, 296, 1045 Carretta, E., Gratton, R. G., \& Sneden, C. 2000, A\&A, 356, 238 Cavallo, R. M., Pilachowski, C. A., \& Rebolo, R. 1997, PASP, 109, 226

Cayrel, R., Depagne, E., Spite, M., et al. 2004, A\&A, 416, 1117 Centurión, M., Molaro, P., Vladilo, G., et al. 2003, A\&A, 403, 55 Chang, R., Hou, J., \& Fu, C. 2000, Chinese Astron. Astrophys., 24, 444

Chen, Y. Q., Nissen, P. E., Zhao, G., Zhang, H. W., \& Benoni, T. 2000, A\&AS, 141, 491

Chiappini, C., Matteucci, F., \& Meynet, G. 2003a, A\&A, 410, 257

Chiappini, C., Romano, D., \& Matteucci, F. 2003b, MNRAS, 339, 63

Chiappini, C., Matteucci, F., \& Ballero, S. K. 2005, A\&A, 437, 429

Clegg, R. E. S., Tomkin, J., \& Lambert, D. L. 1981, ApJ, 250, 262

Daflon, S., \& Cunha, K. 2004, ApJ, 617, 1115

Dahmen, G., Wilson, T. L., \& Matteucci, F. 1995, A\&A, 295, 194

Depagne, E., Hill, V., Spite, M., et al. 2002, A\&A, 390, 187

Diaz, A. I., \& Tosi, M. 1986, A\&A, 158, 60

Drake, J. J., \& Testa, P. 2005, Nature, 436, 525

Ecuvillon, A., Israelian, G., Santos, N. C., et al. 2004, A\&A, 418, 703

Edmunds, M. G., \& Pagel, B. E. J. 1978, MNRAS, 185, 77P

Edvardsson, B., Andersen, J., Gustafsson, B., et al. 1993, A\&A, 275, 101

Esteban, C., Peimbert, M., Torres-Peimbert, S., \& García-Rojas, J. 1999, Rev. Mex. Astron. Astrofis., 35, 65

Esteban, C., García-Rojas, J., Peimbert, M., et al. 2005, ApJ, 618, L95 Ferrini, F., \& Poggianti, B. M. 1993, ApJ, 410, 44

Ferrini, F., Matteucci, F., Pardi, C., \& Penco, U. 1992, ApJ, 387, 138

Ferrini, F., Molla, M., Pardi, M. C., \& Diaz, A. I. 1994, ApJ, 427, 745

Fich, M., \& Silkey, M. 1991, ApJ, 366, 107

Friel, E. D., \& Boesgaard, A. M. 1990, ApJ, 351, 480

Garnett, D. R., Skillman, E. D., Dufour, R. J., et al. 1995, ApJ, 443, 64

Garnett, D. R., Shields, G. A., Peimbert, M., et al. 1999, ApJ, 513, 168 Gavilán, M., Buell, J. F., \& Mollá, M. 2005, A\&A, 432, 861

Gratton, R. G., Sneden, C., Carretta, E., \& Bragaglia, A. 2000, A\&A, 354,169

Grevesse, N., \& Sauval, A. J. 1998, Space Sci. Rev., 85, 161

Gummersbach, C. A., Kaufer, A., Schaefer, D. R., Szeifert, T., \& Wolf, B. 1998, A\&A, 338, 881

Gustafsson, B., Karlsson, T., Olsson, E., Edvardsson, B., \& Ryde, N. 1999, A\&A, 342, 426

Henry, R. B. C., Edmunds, M. G., \& Köppen, J. 2000, ApJ, 541, 660

Holweger, H. 2001, in Joint SOHO/ACE workshop Solar and Galactic Composition, AIP Conf. Proc., 598, 23

Israelian, G., García López, R. J., \& Rebolo, R. 1998, ApJ, 507, 805

Israelian, G., Rebolo, R., García López, R. J., et al. 2001, ApJ, 551, 833

Israelian, G., Ecuvillon, A., Rebolo, R., et al. 2004, A\&A, 421, 649

Iwamoto, K., Brachwitz, F., Nomoto, K., et al. 1999, ApJS, 125, 439

Izotov, Y. I., \& Thuan, T. X. 1999, ApJ, 511, 639

Izotov, Y. I., Thuan, T. X., \& Guseva, N. G. 2005, ApJ

Jørgensen, B. R. 2000, A\&A, 363, 947

Laird, J. B. 1985, ApJ, 289, 556

Lester, D. F., Dinerstein, H. L., Werner, M. W., et al. 1987, ApJ, 320, 573

Mannucci, F., della Valle, M., Panagia, N., et al. 2005, A\&A, 433, 807 Marigo, P. 2001, A\&A, 370, 194

Matteucci, F., \& Greggio, L. 1986, A\&A, 154, 279

Meléndez, J., \& Barbuy, B. 2002, ApJ, 575, 474

Meléndez, J., Barbuy, B., \& Spite, F. 2001, ApJ, 556, 858

Meynet, G., \& Maeder, A. 2002, A\&A, 390, 561

Meyer, D. M., Cardelli, J. A., \& Sofia, U. J. 1997, ApJ, 490, L103
Meyer, D. M., Jura, M., \& Cardelli, J. A. 1998, ApJ, 493, 222

Mishenina, T. V., Korotin, S. A., Klochkova, V. G., \& Panchuk, V. E. 2000, A\&A, 353, 978

Mollá, M., \& Díaz, A. I. 2005, MNRAS, 358, 521

Mollá, M., Vílchez, J. M., Gavilán, M., \& Díaz, A. I. 2006, in preparation

Nissen, P. E. 2002, ArXiv Astrophysics e-prints

Nissen, P. E., Primas, F., Asplund, M., \& Lambert, D. L. 2002, A\&A, 390, 235

Pagel, B. E. J., Edmunds, M. G., Blackwell, D. E., Chun, M. S., \& Smith, G. 1979, MNRAS, 189, 95

Peimbert, M. 1979, in The Large-Scale Characteristics of the Galaxy, IAU Symp., 84, 307

Peimbert, M. 1987, Rev. Mex. Astron. Astrofis., 14, 166

Peimbert, M. 1999, in Chemical Evolution from Zero to High Redshift, 30

Pettini, M., Ellison, S. L., Bergeron, J., \& Petitjean, P. 2002, A\&A, 391, 21

Pilyugin, I. S. 1992, A\&A, 260, 58

Pilyugin, L. S. 1993, A\&A, 277, 42

Pilyugin, L. S., Thuan, T. X., \& Vílchez, J. M. 2003, A\&A, 397, 487

Portinari, L., Chiosi, C., \& Bressan, A. 1998, A\&A, 334, 505

Prantzos, N. 2003, ArXiv Astrophysics e-prints

Prochaska, J. X., Henry, R. B. C., O'Meara, J. M., et al. 2002, PASP, 114,933

Reddy, B. E., Tomkin, J., Lambert, D. L., \& Allende Prieto, C. 2003, MNRAS, 340, 304

Rocha-Pinto, H. J., Maciel, W. J., Scalo, J., \& Flynn, C. 2000a, A\&A, 358,850

Rocha-Pinto, H. J., Scalo, J., Maciel, W. J., \& Flynn, C. 2000b, A\&A, 358, 869

Rolleston, W. R. J., Smartt, S. J., Dufton, P. L., \& Ryans, R. S. I. 2000, A\&A, 363, 537

Ruiz-Lapuente, P., Blinnikov, S., Canal, R., et al. 2000, Mem. Soc. Astron. Ital., 71, 435

Serrano, A., \& Peimbert, M. 1983, Rev. Mex. Astron. Astrofis., 8, 117 Shaver, P. A., McGee, R. X., Newton, L. M., Danks, A. C., \& Pottasch, S. R. 1983, MNRAS, 204, 53

Shi, J. R., Zhao, G., \& Chen, Y. Q. 2002, A\&A, 381, 982

Smartt, S. J., \& Rolleston, W. R. J. 1997, ApJ, 481, L47

Smartt, S. J., Venn, K. A., Dufton, P. L., et al. 2001, A\&A, 367, 86

Smith, V. V., Cunha, K., \& King, J. R. 2001, AJ, 122, 370

Spite, M., Cayrel, R., Plez, B., et al. 2005, A\&A, 430, 655

Timmes, F. X., Woosley, S. E., \& Weaver, T. A. 1995, ApJS, 98, 617

Tomkin, J., \& Lambert, D. L. 1984, ApJ, 279, 220

Tomkin, J., Sneden, C., \& Lambert, D. L. 1986, ApJ, 302, 415

Tomkin, J., Woolf, V. M., Lambert, D. L., \& Lemke, M. 1995, AJ, 109, 2204

Tornambe, A. 1989, MNRAS, 239, 771

Tsamis, Y. G., Barlow, M. J., Liu, X.-W., Danziger, I. J., \& Storey, P. J. 2003, MNRAS, 338, 687

Twarog, B. A. 1980, ApJ, 242, 242

van den Hoek, L. B., \& Groenewegen, M. A. T. 1997, A\&AS, 123, 305

van Zee, L., Salzer, J. J., \& Haynes, M. P. 1998a, ApJ, 497, L1

van Zee, L., Salzer, J. J., \& Haynes, M. P. 1998b, ApJ, 497, L1

Vila Costas, M. B., \& Edmunds, M. G. 1993, MNRAS, 265, 199

Vílchez, J. M., \& Esteban, C. 1996, MNRAS, 280, 720

Wallerstein, G., Iben, I. J., Parker, P., et al. 1997, Rev. Mod. Phys., 69, 995

Westin, J., Sneden, C., Gustafsson, B., \& Cowan, J. J. 2000, ApJ, 530, 783

Woosley, S. E., \& Weaver, T. A. 1995, ApJS, 101, 181 